\title{
Patients' Preference for a Specific Anti-Tumor Necrosis Factor Agent: Korea versus Western
}

\author{
Hyungil Seo* and Byong Duk Ye ${ }^{*,}$ \\ "Department of Gastroenterology and ${ }^{\dagger}$ Inflammatory Bowel Disease Center, Asan Medical Center, University of Ulsan College of Medicine, \\ Seoul, Korea
}

\begin{abstract}
See "Factors Contributing to the Preference of Korean Patients with Crohn's Disease When Selecting an Anti-Tumor Necrosis Factor Agent (CHOICE Study)" by Eun Soo Kim, et al. on page 391-398, Vol. 10. No. 3, 2016
\end{abstract}

The introduction of anti-tumor necrosis factor (TNF) agents for treatment of inflammatory bowel disease (IBD) has changed the treatment paradigm for both Crohn's disease (CD) and ulcerative colitis. ${ }^{1,2}$ Currently, two anti-TNF agents, infliximab (IFX) and adalimumab (ADA), are available in clinical practice in Korea. In general, these anti-TNF agents have demonstrated similar efficacies in the induction and maintenance of clinical remission and responses in moderate to severe $\mathrm{CD} .{ }^{3}$ However, there are no head-to-head comparative trials available to indicate the best options among commercially available anti-TNF agents. Therefore, factors other than efficacy and safety, such as availability, route of administration, patient preference, cost and national guidance, should also be considered when choosing a specific anti-TNF agent. ${ }^{4}$ In fact, selecting a specific antiTNF drug is a typical preference-sensitive decision an individual patient will make in the management of their IBD. Moreover, patients' preferences for specific anti-TNF agents may vary depending on cultural factors. However, data on the preferences of CD patients, especially those in Asia, for anti-TNF agents are very limited.

In this issue of Gut and Liver, Kim et al. ${ }^{5}$ reported the results of the CHOICE study, which investigated the preferences of Korean patients with CD for IFX and ADA and the contributing factors for their preferences. They conducted a prospective questionnaire survey of 189 patients from 10 tertiary referral centers in South Korea. ${ }^{5}$ The authors showed that anti-TNF-naïve CD patients were more likely to favor IFX over ADA. ${ }^{5}$

Several studies have investigated patients' preference for routes of administration of anti-TNF agents in Western settings (Table 1). ${ }^{6-10}$ Interestingly, the findings of Kim et al. ${ }^{5}$ are

Table 1. Results of Studies on Biologic-Naïve Patients' Preference for Anti-Tumor Necrosis Factor Agents

\begin{tabular}{|c|c|c|c|c|c|c|c|}
\hline Author & Study area & Study design & $\begin{array}{l}\text { Study } \\
\text { period }\end{array}$ & $\begin{array}{l}\text { Underlying } \\
\text { disease }\end{array}$ & $\begin{array}{c}\text { No. of } \\
\text { total } \\
\text { patients }\end{array}$ & $\begin{array}{c}\text { No. of } \\
\text { preferring SC } \\
\text { agents (\%) }\end{array}$ & $\begin{array}{c}\text { No. of } \\
\text { preferring IV } \\
\text { agents (\%) }\end{array}$ \\
\hline Kim et al. ${ }^{5}$ & Korea & Hospital-based, multicenter & 2014 & $\mathrm{CD}$ & 189 & 69 (36.5) & $120(63.5)$ \\
\hline Vavricka et al. ${ }^{6}$ & Switzerland & Hospital-based, multicenter & 2008-2009 & $\mathrm{CD}$ & 100 & $64(64.0)^{*}$ & $25(25.0)^{*}$ \\
\hline Williams and Edwards ${ }^{7}$ & United Kingdom & Hospital-based, single center & 2004 & RA & NA & NA $(52.5)^{\dagger}$ & $\mathrm{NA}(17.5)^{\dagger}$ \\
\hline Chilton and Collett $^{8}$ & United Kingdom & Hospital-based, single center & NA $(2008)^{s}$ & RA & 109 & $55(50.5)^{\ddagger}$ & $25(22.9)^{\ddagger}$ \\
\hline Scarpato et al. ${ }^{9}$ & Italy & Hospital-based, multicenter & NA $(2010)^{\mathrm{s}}$ & RA & 802 & $399(49.8)$ & $403(50.2)$ \\
\hline Huynh et al..$^{10}$ & Denmark & Hospital-based, multicenter & NA $(2014)^{\S}$ & RA & 35 & $27(77.1)$ & $8(22.9)$ \\
\hline
\end{tabular}

SC, subcutaneous; IV, intravenous; CD, Crohn's disease; RA, rheumatoid arthritis; NA, not available.

${ }^{*}$ Eleven patients $(11 \%)$ were undecided; ${ }^{\dagger} 30.0 \%$ of patients preferred intramuscular route; ${ }^{\ddagger} 29$ Patients (26.6\%) showed no preference; ${ }^{\circledR}$ Year of study publication.

\section{Correspondence to: Byong Duk Ye}

Department of Gastroenterology and Inflammatory Bowel Disease Center, Asan Medical Center, University of Ulsan College of Medicine, 88 Olympic-ro 43-gil, Songpa-gu, Seoul 05505, Korea

Tel: +82-2-3010-3181, Fax: +82-2-476-0824, E-mail: bdye@amc.seoul.kr pISSN 1976-2283 eISSN 2005-1212 http://dx.doi.org/10.5009/gnl16109

@ This is an Open Access article distributed under the terms of the Creative Commons Attribution Non-Commercial License (http://creativecommons.org/licenses/by-nc/4.0) which permits unrestricted non-commercial use, distribution, and reproduction in any medium, provided the original work is properly cited. 
the complete opposite of the findings from a previous study of Western CD patients. ${ }^{6}$ In the previous study, which was conducted in Switzerland, 100 anti-TNF-naïve CD patients were surveyed, and approximately two-thirds of the patients (64.0\%) preferred subcutaneously delivered drugs (ADA [36.0\%] or certolizumab pegol [28.0\%]) over IFX (25.0\%). ${ }^{6}$ Several studies have investigated the preferences of biologic-naïve patients with rheumatoid arthritis (RA) for biologics. ${ }^{7-10}$ In two studies from the United Kingdom and one from Denmark, RA patients preferred subcutaneous (SC) agents over intravenous (IV) agents. ${ }^{7,8,10}$ However, the largest study of 802 anti-TNF-naïve RA patients from 50 Italian rheumatology centers (the RIVIERA study) revealed similar preferences between SC and IV routes (49.8\% and 50.2\%, respectively). ${ }^{9}$ The varied preferences of study subjects cannot be directly compared between studies because different questionnaires were used. However, the reasons for the patients' preferences for routes of drug administration could help identify the contributing factors to their preferences and thereby further guide the decision-making process.

Kim et al. ${ }^{5}$ showed that logistic factors regarding hospital treatment were the only independent predictive factors for choosing ADA. In the previous study conducted in Switzerland, the ease of administration of anti-TNF therapy and the time spent receiving the therapy were significantly more frequently chosen as deciding factors by CD patients who preferred SC agents in comparison to the group of patients who chose IFX. ${ }^{6}$ For Western RA patients, the most common reasons for choosing ADA or a SC route were not needing to travel to a hospital, ${ }^{8}$ difficulty/discomfort involved in traveling to a hospital, ${ }^{9}$ and a desire to minimize treatment and transportation times. ${ }^{10}$ Therefore, logistic challenges appear to be influential factors for choosing anti-TNF agents for Korean patients with CD and Western patients with RA. In contrast, in the study by Kim et $a l,{ }^{5}$ patients who favored IFX considered a "doctor's presence" as the most important factor when choosing between IFX and ADA. Although the study of CD patients in Switzerland did not include "doctor's presence" as a potential reason for choosing anti-TNF agents, ${ }^{6}$ studies of Western RA patients showed that the most important factors for choosing IFX or an IV route were staff availablity ${ }^{8}$ and the safety of receiving an infusion in the hospital. $^{9,10}$ Therefore, the reasons for selecting specific anti-TNF agents or administration routes among both $\mathrm{CD}$ and RA patients appear to be similar, whether the patients live in more independent Western cultures or in more interdependent Asian cultures. Understanding these behavioral patterns could help physicians guide an individual patient's selection of an appropriate antiTNF agent based on the patient's main concerns (i.e., safety vs convenience).

To understand how a specific anti-TNF agent would be selected in a real-life setting, one more factor that should be considered is the physician's preference. In fact, physician recommendations had more influence on patients' preferences for IBD treatments (steroids, budesonide, immunomodulators, and antiTNF drugs) than routes of administration. ${ }^{6}$ Physician recommendations might be more important for Korean IBD patients due to their relatively interdependent Asian culture, as supported by the results of the study by Kim et al. ${ }^{5}$ The profound impact of physician recommendations emphasizes the importance of physicians having an understanding of their patients' preferences and the factors that contribute to them to guide patients properly in the decision-making process.

In conclusion, the study by Kim et $a l^{5}$ presented the preferences of CD patients in an Asian country for specific anti-TNF agents for the first time. Although factors such as variable costs, accessibility issues, and variable medical facilities were not considered as contributing factors, Kim et al.'s study ${ }^{5}$ suggests that the preference for anti-TNF agents might be heterogeneous in diverse social and cultural environments. To support patient adherence to anti-TNF therapies and improve outcomes, physicians need to understand the factors that have major influences on patients' preferences and perform shared decision-making when selecting anti-TNF agents.

\section{CONFLICTS OF INTEREST}

No potential conflict of interest relevant to this article was reported.

\section{REFERENCES}

1. Lee KM, Lee JM. Crohn's disease in Korea: past, present, and future. Korean J Intern Med 2014;29:558-570.

2. Park SC, Jeen YT. Current and emerging biologics for ulcerative colitis. Gut Liver 2015;9:18-27

3. Peyrin-Biroulet L, Deltenre P, de Suray N, Branche J, Sandborn WJ, Colombel JF. Efficacy and safety of tumor necrosis factor antagonists in Crohn's disease: meta-analysis of placebo-controlled trials. Clin Gastroenterol Hepatol 2008;6:644-653.

4. Dignass A, Van Assche G, Lindsay JO, et al. The second European evidence-based consensus on the diagnosis and management of Crohn's disease: current management. J Crohns Colitis 2010;4:2862.

5. Kim ES, Kim KO, Jang BI, et al. Factors contributing to the preference of Korean patients with Crohn's disease when selecting an anti-tumor necrosis factor agent (CHOICE Study). Gut Liver 2016;10:391-398.

6. Vavricka SR, Bentele N, Scharl M, et al. Systematic assessment of factors influencing preferences of Crohn's disease patients in selecting an anti-tumor necrosis factor agent (CHOOSE TNF TRIAL) Inflamm Bowel Dis 2012;18:1523-1530.

7. Williams EL, Edwards CJ. Patient preferences in choosing antiTNF therapies-R1. Rheumatology (Oxford) 2006;45:1575-1576.

8. Chilton F, Collett RA. Treatment choices, preferences and decisionmaking by patients with rheumatoid arthritis. Musculoskeletal 
Care 2008;6:1-14.

9. Scarpato S, Antivalle M, Favalli EG, et al. Patient preferences in the choice of anti-TNF therapies in rheumatoid arthritis: results from a questionnaire survey (RIVIERA study). Rheumatology (Oxford) 2010;49:289-294.
10. Huynh TK, Ostergaard A, Egsmose C, Madsen OR. Preferences of patients and health professionals for route and frequency of administration of biologic agents in the treatment of rheumatoid arthritis. Patient Prefer Adherence 2014;8:93-99. 